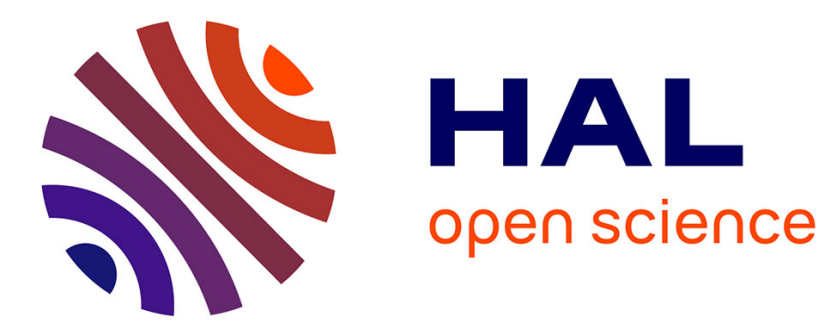

\title{
Valeurs friables d'une forme quadratique et d'une forme linéaire
}

\author{
Armand Lachand
}

\section{To cite this version:}

Armand Lachand. Valeurs friables d'une forme quadratique et d'une forme linéaire. Quarterly Journal of Mathematics, 2014, 66 (1), pp.225-244. 10.1093/qmath/hau029 . hal-01081661

\section{HAL Id: hal-01081661 \\ https://hal.science/hal-01081661}

Submitted on 17 Dec 2014

HAL is a multi-disciplinary open access archive for the deposit and dissemination of scientific research documents, whether they are published or not. The documents may come from teaching and research institutions in France or abroad, or from public or private research centers.
L'archive ouverte pluridisciplinaire HAL, est destinée au dépôt et à la diffusion de documents scientifiques de niveau recherche, publiés ou non, émanant des établissements d'enseignement et de recherche français ou étrangers, des laboratoires publics ou privés. 


\title{
VALEURS FRIABLES D'UNE FORME QUADRATIQUE ET D'UNE FORME LINÉAIRE
}

\author{
ARMAND LACHAND
}

RÉsumé. Given $\mathcal{R}$ a set of integers, $F_{1}\left(X_{1}, X_{2}\right)$ a linear form and $F_{2}\left(X_{1}, X_{2}\right)$ an irreducible quadratic form, we study in this work the cardinality

$$
\#\left\{1 \leqslant n_{1}, n_{2} \leqslant x: F_{1}\left(n_{1}, n_{2}\right) \in \mathcal{R} \text { and } P^{+}\left(F_{2}\left(n_{1}, n_{2}\right)\right) \leqslant y\right\}
$$

where $P^{+}(n)$ denotes the greatest prime factor of an integer $n$. In particular, we give an asymptotic formula for the number of pairs of integers $\left(n_{1}, n_{2}\right)$ in the square $[1, x]^{2}$ such that $F_{1}\left(n_{1}, n_{2}\right)$ and $F_{2}\left(n_{1}, n_{2}\right)$ are both $y$-friable in the range

$$
\exp \left(\frac{\log x(\log \log \log x)^{1+\varepsilon}}{\log \log x}\right) \leqslant y \leqslant x^{2} .
$$

\begin{tabular}{|c|}
\hline 1. Introduction \\
\hline 2. Le $\operatorname{cas} \mathcal{R}=\mathbf{Z}$ \\
\hline 3. Le cas général \\
\hline 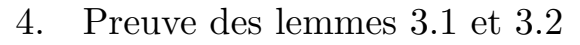 \\
\hline Références \\
\hline
\end{tabular}

\section{TABLE DES MATIÈRES}

\section{INTRODUCTION}

En publiant les Disquisitiones Arithmeticae en 1801, Gauss ouvre la voie à un vaste champ d'étude, à savoir la persistance d'un phénomène arithmétique une fois le champ d'observation restreint aux valeurs d'une forme quadratique. Dans cette direction, Dirichlet établit une formule asymptotique pour le nombre de premiers représentés par une forme quadratique, en lien avec ses travaux sur les corps de nombres quadratiques.

Le problème acquiert une autre dimension si l'on impose une condition supplémentaire à l'une des variables. C'est ainsi que Fouvry et Iwaniec montrent dans [FI97] que, pour tout $A>0$, on a

$$
\sum_{n_{1}^{2}+n_{2}^{2} \leqslant x} \Lambda\left(n_{1}^{2}+n_{2}^{2}\right) \Lambda\left(n_{1}\right)=2 \prod_{p}\left(1-\frac{\chi(p)}{(p-1)(p-\chi(p))}\right) x+O\left(x(\log x)^{-A}\right)
$$

où $\chi$ désigne la caractère non trivial modulo 4 .

Date: 17 décembre 2014. 
Peu de temps après, Friedlander et Iwaniec [FI98b] effectuent le tour de force de restreindre la variable $n_{1}$ dans un ensemble très lacunaire. En particulier, ils démontrent la formule asymptotique

$$
\sum_{n_{1}^{4}+n_{2}^{2} \leqslant x} \Lambda\left(n_{1}^{4}+n_{2}^{2}\right)=\frac{4}{\pi} \int_{0}^{1}\left(1-t^{4}\right)^{1 / 2} \mathrm{~d} t x^{3 / 4}\left(1+O\left(\frac{\log \log x}{\log x}\right)\right) .
$$

Dans ses travaux sur le problème de parité et la conjecture de Chowla, Helfgott étudie un problème analogue en remplaçant la fonction de von Mangoldt $\Lambda$ par la fonction de Liouville $\lambda$. Dans [Hel06, il obtient en particulier que, pour tout convexe $\mathcal{K} \subset[-1,1]$, toute forme linéaire $F_{1}\left(X_{1}, X_{2}\right)$ et toute forme quadratique primitive et irréductible $F_{2}\left(X_{1}, X_{2}\right)$, on a uniformément en $x \geqslant 3$,

$$
\sum_{\left(n_{1}, n_{2}\right) \in \mathcal{K} . x \cap \mathbf{Z}^{2}} \lambda\left(F_{1}\left(n_{1}, n_{2}\right) F_{2}\left(n_{1}, n_{2}\right)\right) \ll \frac{x^{2} \log \log x}{\log x}
$$

où $\mathcal{K} . x:=\left\{\left(a_{1} x, a_{2} x\right):\left(a_{1}, a_{2}\right) \in \mathcal{K}\right\}$.

Un prolongement logique de l'étude consiste à imposer une condition sur chacune des deux variables. Un prototype de cette situation est considéré par La Bretèche et Browning [dlBB10] qui résolvent le problème des diviseurs pour certaines formes quartiques. En particulier, le théorème 2 de dlBB10 stipule que, si $F_{1}$ et $F_{2}$ sont deux formes linéaires non proportionnelles sur $\mathbf{Q}, F_{3}$ est une forme quadratique irréductible et $\mathcal{K} \subset \mathbf{R}^{2}$ est un compact suffisamment régulier tel que $F_{i}(\mathcal{K}) \subset[0,1]$ pour $i \in\{1,2,3\}$, alors il existe une constante $C\left(F_{1}, F_{2}, F_{3}\right)>0$ telle que, pour tout $\varepsilon>0$, on ait, uniformément en $x \geqslant 2$,

$$
\sum_{\left(n_{1}, n_{2}\right) \in \mathcal{K} . x \cap \mathbf{Z}^{2}} \prod_{i=1}^{3} \tau\left(F_{i}\left(n_{1}, n_{2}\right)\right)=C\left(F_{1}, F_{2}, F_{3}\right) \operatorname{Vol}(\mathcal{K}) x^{2}(\log x)^{3}+O\left(x^{2}(\log x)^{2+\varepsilon}\right) .
$$

Dans cet article, on étudie le problème dual de celui considéré dans [FI97, à savoir la représentation des entiers friables par le produit d'une forme linéaire $F_{1}\left(X_{1}, X_{2}\right)$ et d'une forme quadratique $F_{2}\left(X_{1}, X_{2}\right)$. On rappelle qu'un entier $n$ est dit $y$-friable si son plus grand facteur premier, noté $P^{+}(n)$, satisfait $P^{+}(n) \leqslant y$.

La répartition des entiers friables a fait l'objet de nombreux travaux : le lecteur interessé pourra consulter les survols de Hildebrand et Tenenbaum [HT93. ou de Granville Gra08. Par exemple, Hildebrand Hil86 montre que, uniformément dans le domaine

$$
x \geqslant 3, \quad \exp \left((\log \log x)^{5 / 3+\varepsilon}\right) \leqslant y \leqslant x,
$$

on a la formule asymptotique

$$
\Psi(x, y):=\#\left\{1 \leqslant n \leqslant x: P^{+}(n) \leqslant y\right\}=x \rho(u)\left(1+O\left(\frac{\log (u+1)}{\log y}\right)\right)
$$


où, dans cette formule et dans la suite, $u:=\frac{\log x}{\log y}$ et $\rho$ désigne la fonction de Dickman, à savoir l'unique solution de l'équation différentielle aux différences

$$
\begin{cases}\rho(u)=1 & \text { si } 0 \leqslant u \leqslant 1 \\ u \rho^{\prime}(u)+\rho(u-1)=0 & \text { si } u>1\end{cases}
$$

Un raisonnement heuristique consiste à supposer que la friabilité des valeurs des formes $F_{1}$ et $F_{2}$ sont deux événements indépendants, décrits individuellement par le modèle probabiliste suggéré par 1.3. Pour y suffisamment grand, ceci nous amène à conjecturer la formule asymptotique

$$
\#\left\{\left(n_{1}, n_{2}\right) \in \mathcal{K} \cdot x \cap \mathbf{Z}^{2}: P^{+}\left(\left|F_{1}\left(n_{1}, n_{2}\right) F_{2}\left(n_{1}, n_{2}\right)\right|\right) \leqslant y\right\} \underset{x \rightarrow+\infty}{\sim} \operatorname{Vol}(\mathcal{K}) x^{2} \rho(u) \rho(2 u),
$$

sous des hypothèses sur $\mathcal{K}$ et dans un domaine en $(x, y)$ à péciser.

Dans BBDT12, Balog, Blomer, Dartyge et Tenenbaum s'intéressent à l'étude des valeurs friables des formes binaires de degré quelconque. Leur résultat principal est le théorème suivant.

Théorème 1.1 ([BBDT12, Théorème 1$)$. Soient $k \geqslant 1$ et $F_{1}\left(X_{1}, X_{2}\right), \ldots, F_{k}\left(X_{1}, X_{2}\right)$ des formes binaires irréductibles dans $\mathbf{Z}\left[X_{1}, X_{2}\right]$ de degrés respectifs $d_{1} \leqslant \cdots \leqslant d_{k}$. Il existe une constante $u\left(d_{1}, \ldots, d_{k}\right)>1 / d_{k}$ satisfaisant la propriété suivante. Pour tout $\varepsilon>0$ et uniformément en $y \geqslant x^{1 / u\left(d_{1}, \ldots, d_{k}\right)+\varepsilon}$, on a

$$
\#\left\{1 \leqslant n_{1}, n_{2} \leqslant x: P^{+}\left(\left|F_{1}\left(n_{1}, n_{2}\right) \cdots F_{k}\left(n_{1}, n_{2}\right)\right|\right) \leqslant y\right\} \gg_{\varepsilon} x^{2} .
$$

En particulier, si $k=2, F_{1}$ est linéaire et $F_{2}$ est quadratique, la formule (1.5) est valable pour tout $\varepsilon>0$ et uniformément en $y \geqslant x^{\varepsilon}$.

La généralité de ce résultat permet de couvrir le spectre de l'ensemble des formes binaires. En contrepartie et en raison des arguments utilisés, l'information qu'il apporte ne concerne que des domaines bornés en $u$ et ne laisse pas augurer de l'existence d'un équivalent asymptotique. Le théorème suivant - résultat principal de cet article - permet de combler cela : on montre en particulier que la formule (1.4) est valide dans un domaine non borné en $u$.

Théorème 1.2. Soient $F_{1} \in \mathbf{Z}\left[X_{1}, X_{2}\right]$ une forme linéaire primitive, $F_{2} \in \mathbf{Z}\left[X_{1}, X_{2}\right]$ une forme binaire quadratique et irréductible dans $\mathbf{Z}\left[X_{1}, X_{2}\right], \mathcal{K} \subset[0,1]^{2}$ un compact dont le bord est paramétré par un lacet de classe $\mathcal{C}^{1}$ par morceaux. Pour tout $\varepsilon>0$ et dans le domaine

$$
\exp \left(\frac{\log x(\log \log \log x)^{1+\varepsilon}}{\log \log x}\right) \leqslant y \leqslant x^{2},
$$

on a uniformément

$$
\#\left\{\left(n_{1}, n_{2}\right) \in \mathcal{K} . x \cap \mathbf{Z}^{2}: P^{+}\left(\left|F_{1}\left(n_{1}, n_{2}\right) F_{2}\left(n_{1}, n_{2}\right)\right|\right) \leqslant y\right\} \underset{x \rightarrow+\infty}{\sim} \operatorname{Vol}(\mathcal{K}) x^{2} \rho(u) \rho(2 u)
$$

et

$$
\#\left\{\left(n_{1}, n_{2}\right) \in \mathcal{K} . x \cap \mathbf{Z}^{2}: \quad \begin{array}{r}
F_{1}\left(n_{1}, n_{2}\right) \text { premier } \\
\text { et } \quad P^{+}\left(\left|F_{2}\left(n_{1}, n_{2}\right)\right|\right) \leqslant y
\end{array}\right\} \underset{x \rightarrow+\infty}{\sim} \operatorname{Vol}(\mathcal{K}) \frac{x^{2}}{\log x} \rho(2 u) .
$$


Ce résultat constitue un pas supplémentaire vers la preuve de l'algorithme de factorisation du crible quadratique (voir [Pom08]). Une hypothèse importante dans celui-ci consiste en effet à supposer que la formule (1.4) est valide pour

$$
y=\exp \left(2^{-1 / 2+o(1)} \sqrt{\log x \log \log x}\right) .
$$

En raison des méthodes employées dans cet article, notre résultat ne permet pas de couvrir un tel domaine en $(x, y)$, mais constitue un argument de plus en faveur de la pertinence d'une telle heuristique.

L'objet d'étude principal de notre travail concerne plus généralement l'existence de formules asymptotiques pour le cardinal

$$
\Psi(\mathcal{K} . x ; G, y ; \mathcal{R}):=\#\left\{\left(n_{1}, n_{2}\right) \in \mathcal{K} . x \cap \mathbf{Z}^{2}: n_{1} \in \mathcal{R} \text { et } P^{+}\left(\left|G\left(n_{1}, n_{2}\right)\right|\right) \leqslant y\right\}
$$

où $\mathcal{R}$ désigne un ensemble d'entiers et $G\left(X_{1}, X_{2}\right)$ une forme quadratique irréductible dans $\mathbf{Z}\left[X_{1}, X_{2}\right]$. Le résultat suivant, à partir duquel on déduira le théorème 1.2 au paragraphe 3. montre que la $y$-friabilité de $G\left(n_{1}, n_{2}\right)$ et l'appartenance de $n_{1}$ à $\mathcal{R}$ sont essentiellement indépendants.

Théorème 1.3. Soient $G \in \mathbf{Z}\left[X_{1}, X_{2}\right]$ une forme binaire quadratique et irréductible, $\mathcal{K} \subset[0,1]^{2}$ un compact dont le bord est paramétré par un lacet de classe $\mathcal{C}^{1}$ par morceaux et $\mathcal{R}$ un ensemble d'entiers. Pour tout $\varepsilon>0$ et dans le domaine

$$
x \geqslant 3, \quad \exp \left((\log x)^{2 / 5+\varepsilon}\right) \leqslant y \leqslant x,
$$

on a uniformément

$$
\Psi(\mathcal{K} . x ; G, y ; \mathcal{R})=\#\left\{\left(n_{1}, n_{2}\right) \in \mathcal{K} . x \cap \mathbf{Z}^{2}: n_{1} \in \mathcal{R}\right\} \rho(2 u)+O\left(x^{2} \frac{\log \log x}{\log y}\right) .
$$

Si, de plus, il existe $\varepsilon>0$ tel que $\mathcal{R} \subset\left\{P^{-}\left(n_{1}\right)>z\right\}$ avec $z \geqslant \exp \left((\log \log x)^{1+\varepsilon}\right)$, alors on $a$

$$
\Psi(\mathcal{K} . x ; G, y ; \mathcal{R})=\#\left\{\left(n_{1}, n_{2}\right) \in \mathcal{K} . x \cap \mathbf{Z}^{2}: n_{1} \in \mathcal{R}\right\} \rho(2 u)+O\left(\frac{x^{2}}{\log z} \frac{\log \log x}{\log y}\right) .
$$

Étant donné un ensemble de nombres premiers $\mathcal{P}$ et un ensemble d'entiers $\mathcal{R}$ de nature arithmétique, un problème naturel en théorie des nombres réside dans l'estimation du cardinal

$$
S(\mathcal{K} . x ; G, \mathcal{P} ; \mathcal{R}):=\#\left\{\left(n_{1}, n_{2}\right) \in \mathcal{K} . x \cap \mathbf{Z}^{2}: n_{1} \in \mathcal{R} \text { et }\left(p \mid G\left(n_{1}, n_{2}\right) \Rightarrow p \notin \mathcal{P}\right)\right\} .
$$

Le principe d'inclusion-exclusion, à l'origine du développement des méthodes de crible, permet d'obtenir la formule combinatoire

$$
S(\mathcal{K} . x ; G, \mathcal{P} ; \mathcal{R})=\sum_{p \mid d \Rightarrow p \in \mathcal{P}} \mu(d) A_{d}(\mathcal{K} . x, \mathcal{R})
$$

où

$$
A_{d}(\mathcal{K} . x, \mathcal{R}):=\#\left\{\left(n_{1}, n_{2}\right) \in \mathcal{K} . x \cap \mathbf{Z}^{2}: n_{1} \in \mathcal{R}, d \mid G\left(n_{1}, n_{2}\right)\right\} .
$$

$\mathrm{Au}$ vu d'une telle identité, l'étude asymptotique de $S(\mathcal{K} . x ; G, \mathcal{P} ; \mathcal{R})$ se réduit donc a priori à une estimation suffisamment précise de $A_{d}(\mathcal{K} . x, \mathcal{R})$. 
Lorsque $\mathcal{P} \subset\left\{p \leqslant x^{\alpha}\right\}$ avec $\alpha \geqslant 1$, on ne peut pas en général déduire des informations non triviales sur le cardinal $S(\mathcal{K} . x ; G, \mathcal{P} ; \mathbf{Z})$ à partir de la seule connaissance d'estimations de Type I. Ceci est dû au phénomène de parité, étudié en détail par Selberg et Bombieri (voir [Sel52] et [Bom76]). Une manière de surmonter cette difficulté consiste à utiliser l'identité de Vaughan (voir le paragraphe 7 de [FI97] et [FI98a]) pour faire apparaître des sommes bilinéaires, dites de Type II par analogie aux sommes de Type I. Une estimation suffisamment précise de telles sommes constitue le point clé des arguments conduisant aux preuves des résultats précédemment énoncés. À titre d'exemple, Fouvry et Iwaniec montrent que, pour tout $A>0$, on a

$$
\sum_{M_{1}<m_{1} \leqslant 2 M_{1}}\left|\sum_{M_{2}<m_{2} \leqslant 2 M_{2}} \mu\left(m_{2}\right) \delta\left(m_{1}, m_{2}\right)\right| \ll \frac{M_{1} M_{2}}{\left(\log M_{1} M_{2}\right)^{A}}
$$

où

$$
\delta\left(m_{1}, m_{2}\right):= \begin{cases}1 & \text { si } m_{1} m_{2}=n_{1}^{2}+n_{2}^{2} \text { avec } n_{1} \text { premier } \\ 0 & \text { sinon }\end{cases}
$$

et $M_{1}$ et $M_{2}$ sont des paramètres réels.

Notre travail portant sur les entiers friables, on s'intéresse au cas inverse, à savoir $\mathcal{P}:=\{p>y\}$. On retrouve d'une part l'objet d'étude du théorème 1.3 puisque l'on a

$$
\Psi(\mathcal{K} . x ; G, y ; \mathcal{R})=S(\mathcal{K} . x ; G,\{p>y\} ; \mathcal{R}) .
$$

D'autre part, sous des hypothèses convenables concernant la croissance de $u=\frac{\log x}{\log y}$, l'ensemble des entiers $y$-friables est plus dense que celui des entiers criblés et l'on peut donc s'attendre à ce que le phénomène de parité mentionné ci-dessus s'amenuise. Ceci se produit effectivement dans la mesure où $y$ est assez grand ce qui ne nous permet d'obtenir un équivalent asymptotique que dans le domaine (1.6). En vue d'étendre le domaine de validité de la formule, une alternative consisterait à recourir à des estimations similaires à (1.13). Compte tenu de la complexité technique qui en résulterait, nous avons préféré limiter le rang d'application de nos résultats en nous gardant d'introduire les sommes de Type II.

Notre méthode repose essentiellement sur deux étapes distinctes :

- en s'inspirant des estimations de sommes de Type I de [FI97] et [BBDT12], on approche $A_{d}(\mathcal{K} . x, \mathcal{R})$ par $\frac{\gamma(d)}{d^{2}} f_{\mathcal{R}}(x)$ où $f_{\mathcal{R}}$ est une fonction continue et $\gamma$ une fonction multiplicative;

- on estime l'ordre moyen sur les entiers $y$-criblés

$$
T_{\gamma}(x, y):=\sum_{\substack{d \leqslant x \\ P^{-}(d)>y}} \mu(d) \frac{\gamma(d)}{d^{2}}
$$

où $P^{-}(d)$ désigne le plus petit facteur premier de $d$ avec la convention $P^{-}(1)=+\infty$. L'estimation d'une telle somme présente un intérêt intrinsèque et s'inscrit dans une série de travaux comme [All82a], All82b], Hil84] et [Ten90. C'est l'objet de la note [LT] qui accompagne le présent article, réalisée avec Gérald Tenenbaum. 


\section{LE CAS $\mathcal{R}=\mathbf{Z}$}

En guise d'introduction de la méthode, on présente dans ce paragraphe le cas où l'ensemble d'entiers $\mathcal{R}$ est l'ensemble des entiers relatifs $\mathbf{Z}$. La distribution multiplicative des valeurs d'une forme binaire, à travers l'étude de $A_{d}(\mathcal{K} . x, \mathbf{Z})$, a fait l'objet de divers travaux (voir par exemple Gre70, Gre71, [Dan99] et [Mar06]). En vue d'utilisations ultérieures, on rappelle ici le lemme 3.3 de Dan99.

Lemme 2.1 ([Dan99], Lemme 3.3). Soient $G \in \mathbf{Z}\left[X_{1}, X_{2}\right]$ une forme binaire quadratique et irréductible et $\mathcal{K} \subset[0,1]^{2}$ un compact dont le bord est paramétré par un lacet de classe $\mathcal{C}^{1}$ par morceaux. On a, uniformément en $x \geqslant 1$ et $D \geqslant 1$, l'estimation

$$
\sum_{d \leqslant D}\left|A_{d}(\mathcal{K} . x, \mathbf{Z})-\operatorname{Vol}(\mathcal{K}) x^{2} \frac{\gamma(d)}{d^{2}}\right| \ll x \sqrt{D}(\log 2 D)^{250}+D(\log 2 D)^{5}
$$

où

$$
\gamma(d):=\#\left\{1 \leqslant n_{1}, n_{2} \leqslant d: d \mid G\left(n_{1}, n_{2}\right)\right\} .
$$

La fonction arithmétique $\gamma$ introduite dans ce lemme apparaît de manière récurrente dans de nombreux problèmes d'arithmétique liés aux formes binaires. En désignant par $\nu(d)$ le nombre de racines de la congruence

$$
G(1, X) \equiv 0 \quad(\bmod d),
$$

on peut remarquer que, si $p$ est un premier qui ne divise pas $G(0,1)$, alors on a

$$
\gamma(p)=(p-1) \nu(p)+1
$$

Il s'ensuit que le comportement de $\gamma$ est directement lié au corps de nombres $\mathbf{Q}(\omega)$ où $\omega$ désigne une racine du polynôme $G(1, X)$. En effet, un théorème dû à Dedekind [Ded78] assure que, si $p$ ne divise pas l'indice $\left[\mathcal{O}_{\omega}, \mathbf{Z}[\omega]\right]$ où $\mathcal{O}_{\omega}$ désigne l'anneau des entiers de $\mathbf{Q}(\omega)$, alors $\nu(p)$ est égal au nombre d'idéaux premiers $\mathfrak{p}$ de $\mathcal{O}_{\omega}$ dont la norme vaut $p$. De manière générale, le lemme 2.2 de [Mar06] fournit la majoration suivante, uniforme en $p$ premier et $k \geqslant 1$,

$$
\gamma\left(p^{k}\right) \ll_{G} k p^{k}
$$

Soit $\tau \in\left[\frac{1}{\log x}, 2\right]$ un paramètre que l'on précisera en fin d'argument. En utilisant dans l'identité (1.12) l'estimation de la somme de Type I contenue dans le lemme 2.1 avec le niveau de distribution $D=x^{2-\tau}$, il vient

$$
\Psi(\mathcal{K} . x ; G, y ; \mathbf{Z})=\operatorname{Vol}(\mathcal{K}) x^{2} T_{\gamma}\left(x^{2-\tau}, y\right)+O\left(x^{2-\tau / 2}(\log 2 x)^{250}+\sum_{\substack{d>x^{2-\tau} \\ P^{-}(d)>y}} A_{d}(\mathcal{K} . x, \mathbf{Z})\right)
$$

où

$$
T_{\gamma}(t, y):=\sum_{\substack{d \leqslant t \\ P^{-}(d)>y}} \mu(d) \frac{\gamma(d)}{d^{2}}
$$


Écrivons les termes de la sommation du terme d'erreur sous la forme $F\left(n_{1}, n_{2}\right)=m d$ avec $x^{2-\tau}<d \ll x^{2}, P^{-}(d)>y$ et $m \ll x^{\tau}$. On observe ainsi que l'on a

$$
\sum_{\substack{d>x^{2-\tau} \\ P^{-}(d)>y}} A_{d}(\mathcal{K} . x, \mathbf{Z}) \leqslant \sum_{m \ll x^{\tau}} S(\mathcal{K} . x ; m ; Y)
$$

où

$$
S(\mathcal{K} . x ; m ; y):=\#\left\{\left(n_{1}, n_{2}\right) \in \mathcal{K} . x \cap \mathbf{Z}^{2}: m \mid G\left(n_{1}, n_{2}\right) \text { et }\left(p m \mid G\left(n_{1}, n_{2}\right) \Rightarrow p>y\right)\right\} .
$$

On utilise un lemme de crible pour majorer cette dernière quantité. En effet, au vu de (2.1) et compte tenu de la régularité de $\gamma$, les hypothèses $\left(\Omega_{1}\right)$ et $\left(\Omega_{2}(1)\right)$ de [HR74] sont vérifiées et on peut appliquer le théorème 4.1 de [HR74] basé sur le crible de Selberg, pour en déduire l'estimation

$$
\begin{aligned}
& \sum_{m \ll x^{\tau}} S(\mathcal{K} . x ; m ; y) \leqslant \sum_{m \ll x^{\tau}} S\left(\mathcal{K} . x ; m ; \min \left(y, x^{1 / 2}\right)\right) \\
& \ll x^{2} \sum_{m \ll x^{\tau}} \frac{\gamma(m)}{m^{2}} \prod_{\substack{p \leqslant \min \left(y, x^{1 / 2}\right) \\
p \nmid m}}\left(1-\frac{\gamma(p)}{p^{2}}\right) \\
&+\sum_{m \ll x^{1+\tau}} \tau(m)^{3}\left|A_{m}(\mathcal{K} . x, \mathbf{Z})-\operatorname{Vol}(\mathcal{K}) x^{2} \frac{\gamma(m)}{m^{2}}\right| .
\end{aligned}
$$

En utilisant (2.1), 2.2) et le théorème des idéaux premiers, on peut observer la validité des bornes supérieures suivantes, uniformes en $t \geqslant 2$,

$$
\sum_{m \leqslant t} \frac{\gamma(m)}{m^{2}} \prod_{\substack{p \mid m \\ \gamma(p) \neq p^{2}}}\left(1-\frac{\gamma(p)}{p^{2}}\right)^{-1} \ll \prod_{p \leqslant t}\left(1+\frac{\gamma(p)}{p^{2}}\right) \ll \log t
$$

et

$$
\prod_{\substack{p \leqslant t \\ \gamma(p) \neq p^{2}}}\left(1-\frac{\gamma(p)}{p^{2}}\right) \ll(\log t)^{-1} .
$$

Il suit alors de ces estimations que

$$
\begin{aligned}
& \sum_{m \ll x^{\tau}} \frac{\gamma(m)}{m^{2}} \prod_{\substack{p \leqslant \min \left(y, x^{1 / 2}\right) \\
p \nmid m}}\left(1-\frac{\gamma(p)}{p^{2}}\right) \\
\ll & \prod_{\substack{p \leqslant \min \left(y, x^{1 / 2}\right) \\
\gamma(p) \neq p^{2}}}\left(1-\frac{\gamma(p)}{p^{2}}\right) \sum_{m \ll x^{\tau}} \frac{\gamma(m)}{m^{2}} \prod_{\substack{p \mid m \\
\gamma(p) \neq p^{2}}}\left(1-\frac{\gamma(p)}{p^{2}}\right)^{-1} \ll \tau \frac{\log x}{\log y} .
\end{aligned}
$$

Pour traiter la seconde somme de (2.5), on observe tout d'abord que, pour tout $A \geqslant 0$, les estimations (2.1) et (2.2) entraînent la majoration

$$
\sum_{m \ll x^{1+\tau}} \tau(m)^{A} \frac{\gamma(m)}{m^{2}} \ll(\log x)^{2^{A}-1}
$$


tandis que le théorème 1 de dlBB06. permet de montrer que

$$
\sum_{m \ll x^{1+\tau}} \tau(m)^{A} A_{m}(\mathcal{K} . x, \mathbf{Z}) \ll \sum_{\left|n_{1}\right|,\left|n_{2}\right| \ll x} \tau\left(G\left(n_{1}, n_{2}\right)\right)^{A+1} \ll x^{2}(\log x)^{2^{A+1}-1} .
$$

En utilisant l'inégalité de Cauchy-Schwarz, on déduit du lemme 2.1 et de ces deux estimations l'existence d'une constante $c>0$ telle que

$$
\begin{aligned}
\sum_{m \ll x^{1+\tau}} \tau(m)^{3}\left|A_{m}(\mathcal{K} . x, \mathbf{Z})-\operatorname{Vol}(\mathcal{K}) x^{2} \frac{\gamma(m)}{m^{2}}\right| & \\
\ll\left(\sum _ { m \ll x ^ { 1 + \tau } } \tau ( m ) ^ { 6 } \left(A_{m}(\mathcal{K} . x, \mathbf{Z})+\right.\right. & \left.\left.\operatorname{Vol}(\mathcal{K}) x^{2} \frac{\gamma(m)}{m^{2}}\right)\right)^{1 / 2} \\
& \times\left(\sum_{m \ll x^{1+\tau}}\left|A_{m}(\mathcal{K} . x, \mathbf{Z})-\operatorname{Vol}(\mathcal{K}) x^{2} \frac{\gamma(m)}{m^{2}}\right|\right)^{1 / 2}
\end{aligned}
$$

$$
\ll x^{7 / 4+\tau / 4}(\log x)^{c} \text {. }
$$

Il s'ensuit finalement que

$$
\sum_{m \ll x^{\tau}} S(\mathcal{K} . x ; m ; y) \ll \tau \frac{\log x}{\log y} x^{2}+x^{7 / 4+\tau / 4}(\log x)^{c} .
$$

Il reste à étudier la quantité $T_{\gamma}(t, y)$. On remarque tout d'abord que l'estimation 2.1 entraîne que, pour $0<\sigma \leqslant 1$ et uniformément en $p$ premier, on a

$$
\begin{aligned}
& \sum_{k \geqslant 1} p^{-k \sigma}\left|\#\left\{J: N(J)=p^{k}\right\}-\#\left\{J: N(J)=p^{k-1}\right\} \frac{\gamma(p)}{p}\right| \\
& \ll \frac{1}{p^{\sigma+1}}+\sum_{k \geqslant 2} \frac{\#\left\{J: N(J)=p^{k}\right\}}{p^{k \sigma}} \ll \frac{1}{p^{\sigma+1}}+\frac{1}{p^{2 \sigma}} .
\end{aligned}
$$

Il en résulte que la série de Dirichlet $\left(\sum_{n} \mu(n) \frac{\gamma(n)}{n^{s+1}}\right) \zeta_{\mathbf{Q}(\omega)}(s)$ possède le développement en produit eulérien

$$
\prod_{p}\left(1+\sum_{k \geqslant 1} p^{-k s}\left(\#\left\{J: N(J)=p^{k}\right\}-\#\left\{J: N(J)=p^{k-1}\right\} \frac{\gamma(p)}{p}\right)\right)
$$

absolument convergent pour $\Re(s)>\frac{1}{2}$ et qu'un résultat similaire est valable pour la convolution $\left(\sum_{n} \frac{\gamma(n)}{n^{s+1}}\right) \zeta_{\mathbf{Q}(\omega)}(s)^{-1}$.

Compte tenu de ce qui précède, nous pouvons utiliser le théorème principal de [LT], rappelé ci-dessous, pour évaluer la moyenne de la fonction $n \mapsto \mu(n) \frac{\gamma(n)}{n}$ sur les entiers criblés.

Théorème ([LT], Théorème 1.1). Soient $c_{0}>0, \varepsilon>0$ et $\mathbf{K}$ un corps de nombres. Soient $h$ et $\widetilde{h}$ des fonctions arithmétiques telles que $|h(n)| \leqslant \widetilde{h}(n)$ pour tout entier $n$ et les séries de Dirichlet $\left(\sum_{n} h(n) / n^{s}\right) \zeta_{\mathbf{K}}(s)$ et $\left(\sum_{n} \widetilde{h}(n) / n^{s}\right) \zeta_{\mathbf{K}}(s)^{-1}$ soient développables 
en produits eulériens absolument convergents pour $\Re(s) \geqslant 1-c_{0}$. Uniformément dans le domaine

$$
x \geqslant 2, \quad \exp \left\{(\log x)^{2 / 5+\varepsilon}\right\} \leqslant y \leqslant x / 2,
$$

nous avons

$$
\Phi_{h}(x, y):=\sum_{\substack{n \leqslant x \\ P^{-}(n)>y}} h(n)=\left\{1+O\left(\frac{1}{L_{\varepsilon}(y)}\right)\right\} x \int_{0}^{u-1} \frac{\rho^{\prime}(u-v)}{y^{v}} \mathrm{~d} v
$$

où $L_{\varepsilon}(y):=\mathrm{e}^{(\log y)^{3 / 5-\varepsilon}}$. En particulier, nous avons, uniformément dans le domaine $\left.G_{\varepsilon}^{*}\right)$,

$$
\sum_{\substack{n \leqslant x \\ P^{-}(n)>y}} h(n)=\left\{1+O\left(\frac{\log (u+1)}{\log y}\right)\right\} \frac{x \rho^{\prime}(u)+y}{\log y} .
$$

À partir de ce théorème, une sommation d'Abel permet d'obtenir un équivalent pour $T_{\gamma}(x, y)$.

Proposition 2.2. Soit $\varepsilon>0$. Dans le domaine $\left(G_{\varepsilon}\right)$, on a uniformément

$$
T_{\gamma}(x, y)=\rho(u)+O\left(\rho(u) \frac{\log (u+1)}{\log y}+\frac{1}{L_{\varepsilon}(y)}\right) .
$$

Démonstration. On remarque dans un premier temps que, pour $t \in[1, y]$, on a trivialement $\Phi_{n \mapsto \mu(n) \frac{\gamma(n)}{n}}(t, y)=1$. Dans le domaine $y<t<2 y$, on observe à l'aide d'un changement de variables que le théorème des idéaux premiers implique

$$
\begin{aligned}
\Phi_{n \mapsto \mu(n) \frac{\gamma(n)}{n}}(t, y) & =\int_{y}^{t} \frac{1}{\log r} \mathrm{~d} r+O\left(\frac{t}{L_{\varepsilon}(y)}\right) \\
& =t \int_{0}^{\frac{\log t}{\log y}-1} \rho^{\prime}\left(\frac{\log t}{\log y}-v\right) \frac{\mathrm{d} v}{y^{v}}+O\left(\frac{t}{L_{\varepsilon}(y)}\right) .
\end{aligned}
$$

À l'aide de ce qui précède, de la formule 2.10 et d'une sommation d'Abel, on peut donc écrire

$$
\begin{aligned}
T_{\gamma}(x, y) & =\frac{\Phi_{n \mapsto \mu(n) \frac{\gamma(n)}{n}}(x, y)}{x}+\int_{1}^{y} \frac{1}{t^{2}} \mathrm{~d} t+\int_{y}^{x} \frac{\Phi_{n \mapsto \mu(n) \frac{\gamma(n)}{n}}(t, y)}{t^{2}} \mathrm{~d} t . \\
& =\int_{0}^{u-1} \frac{\rho^{\prime}(u-v)}{y^{v}} \mathrm{~d} v+1+\int_{y}^{x} \int_{0}^{\frac{\log t}{\log y}-1} \frac{\rho^{\prime}\left(\frac{\log t}{\log y}-v\right)}{t y^{v}} \mathrm{~d} v \mathrm{~d} t+O\left(\frac{1}{L_{\varepsilon}(y)}\right) \\
& =\int_{0}^{u-1} \frac{\rho^{\prime}(u-v)}{y^{v}} \mathrm{~d} v+1+\log y \int_{0}^{u-1} \int_{v+1}^{u} \frac{\rho^{\prime}(w-v)}{y^{v}} \mathrm{~d} w \mathrm{~d} v+O\left(\frac{1}{L_{\varepsilon}(y)}\right) .
\end{aligned}
$$

On peut majorer la première intégrale en utilisant l'estimation (voir la formule (III.5.82) de [Ten08])

$$
\int_{0}^{u} \frac{\rho^{\prime}(u-v)}{y^{v}} \mathrm{~d} v \ll \rho(u) \frac{\log (u+1)}{\log y} .
$$


Pour la seconde intégrale, on observe à l'aide d'une intégration par parties que l'on a

$$
\begin{aligned}
\log y \int_{0}^{u-1} \int_{v+1}^{u} \frac{\rho^{\prime}(w-v)}{y^{v}} \mathrm{~d} w \mathrm{~d} v & =\log y \int_{0}^{u-1} \frac{\rho(u-v)-1}{y^{v}} \mathrm{~d} v \\
& =\rho(u)-1+O\left(\rho(u) \frac{\log (u+1)}{\log y}\right),
\end{aligned}
$$

ce qui implique le résultat.

Remarque. Compte tenu de la présence de $\frac{1}{L_{\varepsilon}(y)}$ dans le terme d'erreur de 2.12 , on observe que l'équivalent

$$
T_{\gamma}(x, y)=\rho(u)\left(1+O\left(\frac{\log (u+1)}{\log y}\right)\right)
$$

est valide uniformément dans le domaine

$$
\exp \left((\log x)^{5 / 8+\varepsilon}\right) \leqslant y \leqslant x .
$$

Dans la mesure où la formule (III.5.114) de [Ten08] assure que, uniformément pour $u \geqslant 0$ et $0 \leqslant v \leqslant \min \left(u, \frac{1}{\log (u+2)}\right)$, on a

$$
\rho(u-v)=\rho(u)(1+O(v \log (u+1))),
$$

il vient, sous le choix $\tau:=c_{1} \frac{\log \log x}{\log x}$ avec $c_{1}>0$ assez grand et dans le domaine $G_{\varepsilon}$, la formule

$$
T_{\gamma}\left(x^{2-\tau}, y\right)=\rho(2 u)+O\left(\rho(2 u) \log (u+1)\left(\frac{\log \log x}{\log y}+\frac{1}{\log y}\right)+\frac{1}{L_{\varepsilon}(y)}\right) .
$$

On déduit alors de (2.3), 2.4 , 2.9) et 2.14) que l'on a, dans le domaine $\left(G_{\varepsilon}\right)$, la formule

$$
\Psi(\mathcal{K} . x ; G, y ; \mathbf{Z})=\operatorname{Vol}(\mathcal{K}) x^{2} \rho(2 u)+O\left(x^{2} \frac{\log \log x}{\log y}\right) .
$$

Remarque. Dans le cas où $G$ est une forme quadratique irréductible dans $\mathbf{Z}\left[X_{1}, X_{2}\right]$, de discriminant négatif et fondamental, et $\mathcal{K}:=\left\{\left(x_{1}, x_{2}\right): \mid G\left(x_{1}, x_{2} \mid \leqslant 1\right\}\right.$, un développement asymptotique du cardinal $\Psi(\mathcal{K} . x ; G, y ; \mathbf{Z})$ dans le domaine $H_{\varepsilon}$ peut être obtenu en utilisant les résultats de [HTW08].

\section{LE CAS GÉNÉRAL}

On étend à présent l'argument du paragraphe précédent pour des ensembles d'entiers $\mathcal{R}$ quelconques, en conservant le choix $\tau=c_{1} \frac{\log \log x}{\log x}$. Sans perte de généralité, on suppose que $\mathcal{K}$ est un compact de $[0,1]^{2}$ qui vérifie $G(\mathcal{K}) \subset[0,1]$.

Une estimation de sommes de Type I analogue à celle du lemme 2.1 est donnée par le lemme suivant, dont la démonstration fera l'objet du paragraphe 4.

Lemme 3.1. Soient $G \in \mathbf{Z}\left[X_{1}, X_{2}\right]$ une forme binaire primitive, quadratique et irréductible et $\mathcal{K} \subset[0,1]^{2}$ un compact dont le bord est paramétré par un lacet de classe $\mathcal{C}^{1}$ par 
morceaux et satisfaisant $G(\mathcal{K}) \subset[0,1]$. Il existe $c>0$ tel que, uniformément en $x, y \geqslant 1$ et $1 \leqslant D \leqslant x^{2}$, on ait

$$
\begin{aligned}
\sum_{\substack{d \leqslant D \\
P^{-}(d)>y}} \mid A_{d}(\mathcal{K} . x, \mathcal{R})-\frac{\gamma(d)}{d^{2}} \#\left\{\left(n_{1}, n_{2}\right) \in \mathcal{K} . x \cap \mathbf{Z}^{2},\right. & \left.n_{1} \in \mathcal{R}\right\} \mid \ll x^{5 / 3} D^{1 / 6} \log (2 x)^{c} \\
& +\frac{x^{2}}{y}\left(\log (2 x)+\left(\frac{\log (2 x)}{\log (2 y)}\right)^{c}\right) .
\end{aligned}
$$

En utilisant ce lemme dans la formule 1.12 , on obtient l'analogue de (2.3) suivant

$$
\begin{aligned}
\Psi(\mathcal{K} . x ; G, y ; \mathcal{R})= & T_{\gamma}\left(x^{2-\tau}, y\right) \#\left\{\left(n_{1}, n_{2}\right) \in \mathcal{K} . x \cap \mathbf{Z}^{2}, n_{1} \in \mathcal{R}\right\} \\
& +O\left(x^{2-\tau / 6}(\log 2 x)^{c}+\sum_{m \ll x^{\tau}} S(\mathcal{K} . x ; m ; y ; \mathcal{R})\right)
\end{aligned}
$$

dès que $y \geqslant x^{\tau}$, où

$S(\mathcal{K} . x ; m ; y ; \mathcal{R}):=\#\left\{\left(n_{1}, n_{2}\right) \in \mathcal{K} . x \cap \mathbf{Z}^{2}: n_{1} \in \mathcal{R}, m \mid G\left(n_{1}, n_{2}\right),\left(p m \mid G\left(n_{1}, n_{2}\right) \Rightarrow p>y\right)\right\}$

$$
\leqslant S(\mathcal{K} . x ; m ; y) \text {. }
$$

En utilisant les estimations (2.9) et (2.14), on en déduit la formule asymptotique (1.10).

Supposons à présent que $\mathcal{R} \subset\left\{P^{-}\left(n_{1}\right)>z\right\}$ où $z \geqslant \exp \left((\log \log x)^{1+\varepsilon}\right)$. On peut alors remplacer (3.1) par l'inégalité

$$
S(\mathcal{K} . x ; m ; y ; \mathcal{R}) \leqslant S\left(\mathcal{K} . x ; m ; y ;\left\{P^{-}\left(n_{1}\right)>z\right\}\right) .
$$

Nous démontrerons au paragraphe 4 le lemme suivant, qui peut être vu comme un analogue du lemme 2.1 au cas où $\mathcal{R} \subset\left\{P^{-}\left(n_{1}\right)>z\right\}$.

Lemme 3.2. Soient $B>0, G \in \mathbf{Z}\left[X_{1}, X_{2}\right]$ une forme binaire primitive, quadratique et irréductible et $\mathcal{K} \subset[0,1]^{2}$ un compact dont le bord est paramétré par un lacet de classe $\mathcal{C}^{1}$ par morceaux et satisfaisant $G(\mathcal{K}) \subset[0,1]$. Il existe $c(B)>0$ tel que, uniformément en $x, z \geqslant 1,1 \leqslant D \leqslant x^{2}$ et $\mathcal{R} \subset\left\{n_{1}: P^{-}\left(n_{1}\right)>z\right\}$, on ait

$$
\sum_{d \leqslant D} \tau(d)^{B}\left|A_{d}(\mathcal{K} . x, \mathcal{R})-\frac{\nu(d)}{d} \#\left\{\left(n_{1}, n_{2}\right) \in \mathcal{K} . x \cap \mathbf{Z}^{2}, n_{1} \in \mathcal{R}\right\}\right| \ll\left(x^{5 / 3} D^{1 / 6}+\frac{x^{2}}{z}\right) \log (2 x)^{c(B)} .
$$

On peut alors reproduire l'argument de crible détaillé dans le paragraphe précédent qui a conduit à l'estimation (2.9) en remplaçant l'utilisation du lemme 2.1 par le lemme 3.2. Compte tenu des hypothèses sur $z$, il s'ensuit que

$$
\sum_{m \ll x^{\tau}} S\left(\mathcal{K} . x ; m ; y ;\left\{P^{-}\left(n_{1}\right)>z\right\}\right) \ll \tau \frac{\log x}{\log y} \frac{x^{2}}{\log z},
$$

ce qui permet d'obtenir 1.11.

On conclut ce paragraphe en montrant comment déduire le théorème 1.2 à partir du théorème 1.3 . 
Preuve que le théorème 1.2 implique le théorème 1.3 . Puisque la forme linéaire $F_{1}$ est primitive, écrivons-la sous la forme $F_{1}\left(X_{1}, X_{2}\right)=a_{11} X_{1}+a_{12} X_{2}$ avec $\left(a_{11}, a_{12}\right)=1$. Le théorème de Bachet-Bézout assure alors l'existence de deux entiers $a_{21}$ et $a_{22}$ tels que $\left|\begin{array}{ll}a_{11} & a_{12} \\ a_{21} & a_{22}\end{array}\right|=1$. Sous l'effet du changement de variables $\left(\begin{array}{l}n_{1}^{\prime} \\ n_{2}^{\prime}\end{array}\right)=A\left(\begin{array}{l}n_{1} \\ n_{2}\end{array}\right)$ où l'on a posé $A:=\left(\begin{array}{ll}a_{11} & a_{12} \\ a_{21} & a_{22}\end{array}\right) \in S L_{2}(\mathbf{Z})$, on remarque que l'on a l'identité

$\#\left\{\left(n_{1}, n_{2}\right) \in \mathcal{K} . x \cap \mathbf{Z}^{2}: F_{1}\left(n_{1}, n_{2}\right) \in \mathcal{R}\right.$ et $\left.P^{+}\left(\left|F_{2}\left(n_{1}, n_{2}\right)\right|\right) \leqslant y\right\}=\Psi(A(\mathcal{K}) . X ; G, y ; \mathcal{R})$

où $G\left(X_{1}, X_{2}\right):=F_{2}\left(A^{-1}\left(X_{1}, X_{2}\right)\right)$ est quadratique, primitive et irréductible.

En prenant $\mathcal{R}$ l'ensemble des entiers $y$-friables dans $(1.10)$, on peut utiliser l'estimation de Hildebrand $\sqrt{1.3}$ pour évaluer $\#\left\{\left(n_{1}, n_{2}\right) \in A(\mathcal{K}) \cdot x \cap \mathbf{Z}^{2}: P^{+}\left(n_{1}\right) \leqslant y\right\}$. Pour ce faire, on introduit la fonction $g$ qui à tout réel $x_{1}$ associe la mesure de l'ensemble $\left\{x_{2} \in \mathbf{R}:\left(x_{1}, x_{2}\right) \in A(\mathcal{K}) \cdot x\right\}$. Compte tenu des hypothèses sur le bord de $\mathcal{K}$, on peut écrire $g$ comme la somme de $O_{\mathcal{K}}(1)$ fonctions continues, de classe $\mathcal{C}^{1}$ par morceaux. Par suite, quitte à effectuer une homothétie, on peut supposer sans perte de généralité que $g$ a pour support $[0, x]$, intervalle sur lequel elle est de classe $\mathcal{C}^{1}$. En utilisant une sommation d'Abel, on obtient ainsi

$$
\begin{aligned}
& \#\left\{\left(n_{1}, n_{2}\right) \in A(\mathcal{K}) \cdot x \cap \mathbf{Z}^{2}: P^{+}\left(n_{1}\right) \leqslant y\right\} \\
= & \sum_{\substack{x /(\log x)^{2}<n_{1} \leqslant x \\
P^{+}\left(n_{1}\right) \leqslant y}}\left(g\left(n_{1}\right)+O(1)\right)+O\left(\frac{x^{2}}{(\log x)^{2}}\right) \\
= & g\left(x^{-}\right) \Psi(x, y)-\int_{x /(\log x)^{2}}^{x} \Psi(t, y) g^{\prime}(t) \mathrm{d} t+O\left(\frac{x^{2}}{(\log x)^{2}}\right) \\
= & x g\left(x^{-}\right) \rho(u)-\int_{x /(\log x)^{2}}^{x} t \rho\left(\frac{\log t}{\log y}\right) g^{\prime}(t) \mathrm{d} t\left(1+O\left(\frac{\log (u+1)}{\log y}\right)\right)+O\left(\frac{x^{2}}{(\log x)^{2}}\right) .
\end{aligned}
$$

La formule (2.13) assure que, dans le domaine (1.6), on a

$$
\rho\left(u-\frac{\log (2 \log x)}{\log y}\right)=\rho(u)\left(1+O\left(\frac{\log (u+1) \log \log x}{\log y}\right)\right) .
$$

Puisque $\rho$ est décroissante, il suit finalement que

$$
\begin{aligned}
& \#\left\{\left(n_{1}, n_{2}\right) \in A(\mathcal{K}) \cdot x \cap \mathbf{Z}^{2}: P^{+}\left(n_{1}\right) \leqslant y\right\} \\
= & \rho(u)\left(x g\left(x^{-}\right)-\int_{x /(\log x)^{2}}^{x} t g^{\prime}(t) \mathrm{d} t\right)\left(1+O\left(\frac{\log (u+1) \log \log x}{\log y}\right)\right)+O\left(\frac{x^{2}}{(\log x)^{2}}\right) \\
= & \rho(u) \operatorname{Vol}(A(\mathcal{K})) x^{2}+O\left(x^{2} \rho(u) \frac{\log (u+1) \log \log x}{\log y}+\frac{x^{2}}{(\log x)^{2}}\right) .
\end{aligned}
$$


Dans la mesure où $\operatorname{Vol}(A(\mathcal{K}))=\operatorname{Vol}(\mathcal{K})$, on déduit de la formule 3.2 et du théorème 1.3 que l'on a

$$
\begin{aligned}
\#\left\{\left(n_{1}, n_{2}\right) \in \mathcal{K} . x \cap \mathbf{Z}^{2}: P^{+}\left(\left|F_{1}\left(n_{1}, n_{2}\right) F_{2}\left(n_{1}, n_{2}\right)\right|\right) \leqslant y\right\}= & \operatorname{Vol}(\mathcal{K}) x^{2} \rho(2 u) \\
& +O\left(x^{2} \frac{\log \log x}{\log y}\right) .
\end{aligned}
$$

Le terme d'erreur reste finalement négligeable tant que $(x, y)$ parcourt le domaine 1.6 , au vu de l'estimation $\rho(u)^{-1}=\exp (O(u \log (u+1)))$.

Un raisonnement analogue, basé sur l'utilisation du théorème des nombres premiers et de (1.11) permet de déduire la formule 1.8 portant sur les valeurs de $F_{1}$ premières.

\section{Preuve des lemmes 3.1 et 3.2}

Dans cette partie, on étudie le cardinal $A_{d}(\mathcal{K} . x, \mathcal{R})$, en vue d'établir les lemmes 3.1 et 3.2. Pour des raisons techniques qui apparaîtront au cours de l'argument, on s'intéresse dans un premier temps aux cardinaux

$$
A_{d}^{(1)}(\mathcal{K} . x, \mathcal{R}):=\#\left\{\left(n_{1}, n_{2}\right) \in \mathcal{K} . x \cap \mathbf{Z}^{2}: n_{1} \in \mathcal{R},\left(n_{1}, d\right)=1 \text { et } d \mid G\left(n_{1}, n_{2}\right)\right\} .
$$

Lemme 4.1. Soient $B>0, G \in \mathbf{Z}\left[X_{1}, X_{2}\right]$ une forme binaire primitive, quadratique et irréductible, $\mathcal{K} \subset[0,1]^{2}$ un compact dont le bord est paramétré par un lacet de classe $\mathcal{C}^{1}$ par morceaux et satisfaisant $G(\mathcal{K}) \subset[0,1]$ et $\mathcal{R}$ un ensemble d'entiers. Il existe $c(B)>0$ tel que, uniformément en $1 \leqslant D \leqslant x^{2}$, on ait

$$
\begin{aligned}
& \quad \sum_{d \leqslant D} \tau(d)^{B}\left|A_{d}^{(1)}(\mathcal{K} . x, \mathcal{R})-\frac{\nu(d)}{d} \#\left\{\left(n_{1}, n_{2}\right) \in \mathcal{K} . x \cap \mathbf{Z}^{2}, n_{1} \in \mathcal{R},\left(n_{1}, d\right)=1\right\}\right| \\
& \ll x^{5 / 3} D^{1 / 6} \log (2 x)^{c(B)} .
\end{aligned}
$$

La preuve de ce lemme consiste essentiellement à adapter les parties 2 et 3 de [FI97] et le chapitre 20.3 de [FI10] où un analogue de ce résultat est établi pour la forme quadratique $F\left(X_{1}, X_{2}\right)=X_{1}^{2}+X_{2}^{2}$. Une généralisation à des formes quadratiques quelconques s'appuie en particulier sur les parties 5.2 et 5.3 de [BBDT12] dans lesquelles Balog et al. obtiennent essentiellement le lemme 4.1 avec $x^{\varepsilon}$ au lieu de $\log (2 x)$.

Démonstration. Soit $\Delta \in[1, x]$ un paramètre dont le choix sera rendu explicite au cours de la preuve. En effectuant la convolution de l'indicatrice de $\mathcal{K} . x$ et d'une suite régularisante, on montre l'existence d'une fonction lisse $\chi_{\mathcal{K} . x}$ à valeurs dans $[0,1]$ telle que

$$
\begin{aligned}
& \operatorname{supp} \chi_{\mathcal{K} . x} \subset \mathcal{K} . x, \\
& \chi_{\mathcal{K} . x}\left(n_{1}, n_{2}\right)=1 \text { si } d\left(\left(n_{1}, n_{2}\right), \mathbf{R}^{2} \backslash \mathcal{K} . x\right)>\Delta, \\
& \frac{\partial^{j} \chi_{\mathcal{K} . x}}{\partial s^{j}}\left(n_{1}, n_{2}\right) \ll \Delta^{-j} \text { pour } j \in\{0,1,2\} .
\end{aligned}
$$

On approche $A_{d}^{(1)}(\mathcal{K} . x, \mathcal{R})$ par la quantité

$$
A_{d}^{(1)}\left(\chi_{\mathcal{K} . x}, \mathcal{R}\right):=\sum_{\substack{\left(n_{1}, n_{2}\right) \in \mathcal{R} \times \mathbf{Z} \\\left(n_{1}, d\right)=1 \\ d \mid G\left(n_{1}, n_{2}\right)}} \chi_{\mathcal{K} . x}\left(n_{1}, n_{2}\right),
$$


l'apparition de $\chi_{\mathcal{K} . x}$ en lieu et place de l'indicatrice de $\mathcal{K} . x$ fournissant un cadre propice à l'utilisation de l'analyse de Fourier.

Le terme d'erreur qui apparaît sous l'effet d'une telle démarche est négligeable. En effet, d'après l'inégalité de Cauchy-Schwarz et les hypothèses de régularité faites sur le bord de $\mathcal{K}$, on a

$$
\begin{aligned}
\sum_{d \leqslant D}\left|A_{d}^{(1)}(\mathcal{K} . x, \mathcal{R})-A_{d}^{(1)}\left(\chi_{\mathcal{K} . x}, \mathcal{R}\right)\right| & \ll\left(\sum_{\left|n_{1}\right|,\left|n_{2}\right| \leqslant x} \tau\left(G\left(n_{1}, n_{2}\right)\right)^{2}\right)^{\frac{1}{2}} \\
& \times \#\left\{\left(n_{1}, n_{2}\right) \in \mathcal{K} . x \cap \mathbf{Z}^{2}: \chi_{\mathcal{K} . x}\left(n_{1}, n_{2}\right) \neq 1\right\}^{\frac{1}{2}} .
\end{aligned}
$$

Dans la mesure où la fonction $\tau^{2}$ satisfait les hypothèses du théorème 1 de dlBB06, on peut estimer la somme du membre de droite. Compte tenu de la régularité du bord de $\mathcal{K}$, on en déduit ainsi que

$$
\sum_{d \leqslant D}\left|A_{d}^{(1)}(\mathcal{K} . x, \mathcal{R})-A_{d}^{(1)}\left(\chi_{\mathcal{K} . x}, \mathcal{R}\right)\right| \ll x^{\frac{3}{2}} \Delta^{\frac{1}{2}} \prod_{p \leqslant x}\left(1+3 \frac{\gamma^{*}(p)}{p}\right)^{\frac{1}{2}}
$$

où

$$
\gamma^{*}(p)=\frac{1}{p-1} \#\left\{0 \leqslant n_{1}, n_{2}<p:\left(n_{1}, n_{2}, p\right)=1 \text { et } G\left(n_{1}, n_{2}\right)=p\right\} .
$$

Puisque $\gamma^{*}(p)=\nu(p)+O(1 / p)$ (formule (1.6) de [lBB06]), il suit finalement que

$$
\sum_{d \leqslant D}\left|A_{d}^{(1)}(\mathcal{K} . x, \mathcal{R})-A_{d}^{(1)}\left(\chi_{\mathcal{K} . x}, \mathcal{R}\right)\right| \ll x^{\frac{3}{2}} \Delta^{\frac{1}{2}}(\log x)^{3 / 2} .
$$

On étudie à présent les quantités $A_{d}^{(1)}\left(\chi_{\mathcal{K} . x}, \mathcal{R}\right)$. En utilisant la formule sommatoire de Poisson, on obtient l'identité

$$
A_{d}^{(1)}\left(\chi_{\mathcal{K} . x}, \mathcal{R}\right)=\frac{1}{d} \sum_{\substack{n_{1} \in \mathcal{R} \\\left(n_{1}, d\right)=1}} \sum_{k \in \mathbf{Z}} \gamma_{k, n_{1}}(d) \int_{-\infty}^{+\infty} \chi_{\mathcal{K} . x}\left(n_{1}, t\right) \mathrm{e}\left(-\frac{t k}{d}\right) \mathrm{d} t
$$

où $\mathrm{e}(t):=\exp (2 i \pi t)$ et

$$
\gamma_{k, n_{1}}(d)=\sum_{\substack{n_{2} \bmod d \\ d \mid G\left(n_{1}, n_{2}\right)}} \mathrm{e}\left(\frac{n_{2} k}{d}\right) .
$$

Au vu de la condition $\left(n_{1}, d\right)=1$, on remarque que l'on a notamment

$$
\gamma_{k, n_{1}}(d)=\sum_{\substack{\nu \bmod d \\ d \mid G(1, \nu)}} \mathrm{e}\left(\frac{\nu k n_{1}}{d}\right)
$$

ce qui entraîne l'identité $\gamma_{0, n_{1}}(d)=\nu(d)$.

Le terme principal, provenant de la fréquence $k=0$, est donné par la quantité

$$
\frac{\nu(d)}{d} \sum_{\substack{n_{1} \in \mathcal{R} \\\left(n_{1}, d\right)=1}} \int_{-\infty}^{+\infty} \chi_{\mathcal{K} . x}\left(n_{1}, t\right) \mathrm{d} t
$$


Par suite, on retrouve le terme principal attendu dans l'énoncé du lemme en remarquant que

$$
\begin{aligned}
& \sum_{d \leqslant D} \frac{\nu(d)}{d} \sum_{\substack{n_{1} \in \mathcal{R} \\
\left(n_{1}, d\right)=1}}\left|\int_{-\infty}^{+\infty} \chi \mathcal{K} . x\left(n_{1}, t\right) \mathrm{d} t-\#\left\{n_{2}:\left(n_{1}, n_{2}\right) \in \mathcal{K} . x \cap \mathbf{Z}^{2}\right\}\right| \\
& \ll \sum_{d \leqslant D} \frac{\nu(d)}{d} \sum_{n_{1} \in \mathcal{R}}\left(\#\left\{n_{2}:\left(n_{1}, n_{2}\right) \in \mathcal{K} . x \cap \mathbf{Z}^{2}: \chi_{\mathcal{K} . x}\left(n_{1}, n_{2}\right) \neq 1\right\}+O(1)\right) \\
& \ll \Delta x \log (2 D) .
\end{aligned}
$$

Le cas des fréquences non nulles fait appel à une inégalité de grand crible, essentiellement énoncée dans la proposition 3 de BBDT12. On précise dans le lemme qui suit ce résultat en remplaçant le terme $(D K R)^{\delta}$ obtenu dans BBDT12 par $\log (2 D)$, modification qui permet de choisir un niveau de distribution $D=X^{2-\tau}$.

Lemme 4.2. On a, uniformément pour tous entiers $D, K, x \geqslant 1$ et toute suite de complexes $\left(\xi_{k, n}\right)$, l'inégalité de grand crible

$$
\sum_{d \leqslant D}\left|\sum_{k \leqslant K} \sum_{\substack{n \leqslant x \\(n, d)=1}} \xi_{k, n} \gamma_{k, n}(d)\right| \ll D^{1 / 2}(D+K x)^{1 / 2}\left(\sum_{k, n}\left|\xi_{k, n}\right|^{2} \tau(k n)\right)^{1 / 2} \log (2 D) .
$$

Démonstration. D'après la preuve de la proposition 3 de [BBDT12], on a, pour toute suite de complexes $\left(\xi_{n}\right)$, l'estimation

$$
\sum_{D \leqslant d \leqslant 2 D} \sum_{G(1, \nu) \equiv 0}\left|\sum_{n \leqslant x} \xi_{n} \mathrm{e}\left(\frac{\nu n}{d}\right)\right|^{2} \ll(D+x) \sum_{n \leqslant x}\left|\xi_{n}\right|^{2},
$$

ce qui constitue une généralisation du lemme 2 de [FI97] à des polynômes quadratiques irréductibles quelconques. En reproduisant le raisonnement développé à la page 253 de [FI97, il s'ensuit alors que

$$
\begin{aligned}
& \sum_{d \leqslant D} \sum_{G(1, \nu) \equiv 0}\left|\sum_{(\bmod d)} \xi_{k, n} \mathrm{e}\left(\frac{\nu k n}{d}\right)\right| \\
& \ll \sum_{b \leqslant D} \nu(b) \sum_{d \leqslant D / b} \sum_{\substack{k, n \leqslant x \\
(n, d)=1}}\left|\sum_{k \leqslant K, n \leqslant 0} \xi_{k, n b} \mathrm{e}\left(\frac{\nu k n}{d}\right)\right| \\
& \ll D^{1 / 2}(D+K x)^{1 / 2}\left(\sum_{k, n}\left|\xi_{k, n}\right|^{2} \tau(k n)\right)^{1 / 2} \log (2 D)
\end{aligned}
$$

ce qui implique le résultat attendu. 
Reprenons la preuve du lemme 4.1. En effectuant le changement de variable $w=\frac{t k}{x}$ afin de séparer les variables $k$ et $d$ dans l'intégrale, on obtient

$$
\int_{-\infty}^{+\infty} \chi_{\mathcal{K} . x}\left(n_{1}, t\right) \mathrm{e}\left(-\frac{t k}{d}\right) \mathrm{d} t=\frac{x}{k} \int_{-K}^{K} \chi_{\mathcal{K} . x}\left(n_{1}, \frac{w x}{k}\right) \text { e }\left(-\frac{w x}{d}\right) \mathrm{d} w .
$$

On déduit alors du lemme 4.2 que

$$
\begin{aligned}
& \sum_{D<d \leqslant 2 D}\left|\sum_{\substack{n_{1} \in \mathcal{R} \\
\left(n_{1}, d\right)=1}} \sum_{K<k \leqslant 2 K} \frac{x}{d k} \gamma_{k, n_{1}}(d) \int_{-K}^{K} \chi_{\mathcal{K} . x}\left(n_{1}, \frac{w x}{k}\right) \mathrm{e}\left(-\frac{w x}{d}\right) \mathrm{d} w\right| \\
& \leqslant \sum_{D<d \leqslant 2 D} \int_{-K}^{K}\left|\sum_{\substack{n_{1} \in \mathcal{R} \\
\left(n_{1}, d\right)=1}} \sum_{K<k \leqslant 2 K} \frac{x}{d k} \gamma_{k, n_{1}}(d) \chi_{\mathcal{K} . x}\left(n_{1}, \frac{w x}{k}\right)\right| \mathrm{d} w \\
& \ll x D^{-\frac{1}{2}}(D+K x)^{\frac{1}{2}} \log (2 D)\left(\sum_{k \leqslant 2 K, n_{1} \ll x} \tau\left(k n_{1}\right)\right)^{1 / 2} \\
& \ll x^{\frac{3}{2}} D^{-\frac{1}{2}} K^{\frac{1}{2}}(D+K x)^{\frac{1}{2}} \log (2 D K x)^{5 / 2} .
\end{aligned}
$$

Pour les fréquences $k$ grandes, une telle estimation n'est pas convenable. En intégrant deux fois par parties, on obtient la formule

$$
\int_{-K}^{K} \chi \mathcal{K} \cdot x\left(n_{1}, \frac{w x}{k}\right) \text { e }\left(-\frac{w x}{d}\right) \mathrm{d} w=\frac{1}{4 \pi^{2}} \frac{d^{2}}{k^{2}} \int_{-K}^{K} \frac{\partial^{2} \chi \mathcal{K} \cdot x}{\partial s^{2}}\left(n_{1}, \frac{w x}{k}\right) \text { e }\left(-\frac{w x}{d}\right) \mathrm{d} w .
$$

En utilisant là encore l'inégalité du grand crible du lemme 4.2, on en déduit

$$
\begin{aligned}
& \sum_{D<d \leqslant 2 D}\left|\sum_{\substack{n_{1} \in \mathcal{R} \\
\left(n_{1}, d\right)=1}} \sum_{K<k \leqslant 2 K} \frac{x}{d k} \gamma_{k, n_{1}}(d) \int_{-K}^{K} \chi_{\mathcal{K} \cdot x}\left(n_{1}, \frac{w x}{k}\right) \mathrm{e}\left(-\frac{w x}{d}\right) \mathrm{d} w\right| \\
& \leqslant \frac{x}{4 \pi^{2}} \sum_{D<d \leqslant 2 D} \int_{-K}^{K}\left|\sum_{\substack{n_{1} \in \mathcal{R} \\
\left(n_{1}, d\right)=1}} \sum_{K<k \leqslant 2 K} \frac{d}{k^{3}} \gamma_{k, n_{1}}(d) \frac{\partial^{2} \chi \mathcal{K} \cdot x}{\partial s^{2}}\left(n_{1}, \frac{w x}{k}\right)\right| \mathrm{d} w \\
& \ll x D^{\frac{3}{2}} K^{-2}(D+K x)^{\frac{1}{2}} \Delta^{-2} \log (2 D)\left(\sum_{k \leqslant 2 K, n_{1} \ll x} \tau\left(k n_{1}\right)\right)^{1 / 2} \\
& \ll x^{\frac{3}{2}} D^{\frac{3}{2}} K^{-\frac{3}{2}}(D+K x)^{\frac{1}{2}} \Delta^{-2} \log (2 D K x)^{5 / 2} .
\end{aligned}
$$


En utilisant (4.7) si $K \leqslant D \Delta^{-1}$ et 4.8 sinon et en sommant sur les puissances de 2 issues des découpages dyadiques en $d$ et $k$, on en déduit

$$
\begin{aligned}
& \sum_{d \leqslant D}\left|\sum_{\substack{n_{1} \in \mathcal{R} \\
\left(n_{1}, d\right)=1}} \sum_{k \neq 0} \frac{x}{d k} \gamma_{k, n_{1}}(d) \int_{-K}^{K} \chi \mathcal{K} \cdot x\left(n_{1}, \frac{w x}{k}\right) \mathrm{e}\left(-\frac{w x}{k}\right)\right| \mathrm{d} w \\
& \ll\left(x^{3 / 2} D^{1 / 2} \Delta^{-1 / 2}+x^{2} D^{1 / 2} \Delta^{-1}\right) \log (2 x)^{5 / 2} .
\end{aligned}
$$

En choisissant $\Delta=x^{\frac{1}{3}} D^{\frac{1}{3}}$ et en collectant les termes d'erreur 4.3 , 4.5) et 4.9 , on en déduit que

$$
\sum_{d \leqslant D}\left|A_{d}^{(1)}(\mathcal{K} . x, \mathcal{R})-\frac{\nu(d)}{d} \#\left\{\left(n_{1}, n_{2}\right) \in \mathcal{K} . x \cap \mathbf{Z}^{2}, n_{1} \in \mathcal{R},\left(n_{1}, d\right)=1\right\}\right| \ll x^{5 / 3} D^{1 / 6} \log (2 x)^{5 / 2} .
$$

On peut alors utiliser l'inégalité de Cauchy-Schwarz et reproduire l'argument qui avait conduit à (2.8) pour en déduire le lemme.

Preuve des lemmes 3.1 et 3.2. À partir de 2.1), on observe que

$$
\frac{\gamma(p)}{p^{2}}=\frac{\nu(p)}{p}+O\left(\frac{1}{p^{2}}\right)
$$

d'où l'on peut déduire que

$$
\sum_{\substack{d \leqslant x^{2} \\ P^{-}(d)>y}}\left|\frac{\nu(d)}{d}-\frac{\gamma(d)}{d^{2}}\right| \ll \frac{1}{y} \sum_{\substack{d \leqslant x^{2} \\ P^{-}(d)>y}} \mu^{2}(d) \frac{\tau(d)^{c}}{d} \ll \frac{1}{y} \sum_{k \geqslant 0} \frac{1}{k !}\left(\sum_{y<p \leqslant x^{2}} \frac{2^{c}}{p}\right)^{k} \ll \frac{1}{y}\left(\frac{\log (2 x)}{\log (2 y)}\right)^{2^{c}}
$$

pour une constante $c>0$. Au vu du lemme 4.1, il suffit donc de montrer que, pour tout $B \geqslant 0$, on a

$$
\begin{gathered}
\sum_{d \leqslant x^{2}} \tau(d)^{B} \mid \#\left\{\left(n_{1}, n_{2}\right) \in \mathcal{K} . x \cap \mathbf{Z}^{2}:\left(n_{1}, d\right) \neq 1, P^{-}\left(\left(n_{1}, d\right)\right)>y \text { et } d \mid G\left(n_{1}, n_{2}\right)\right\} \\
-\frac{\nu(d)}{d} \#\left\{\left(n_{1}, n_{2}\right) \in \mathcal{K} . x \cap \mathbf{Z}^{2}:\left(n_{1}, d\right) \neq 1, P^{-}\left(\left(n_{1}, d\right)\right)>y\right\} \mid \ll \frac{x^{2}}{y} \log (2 x)^{c(B)}
\end{gathered}
$$

pour une certaine constante $c(B)$. On remarque d'une part que l'on a

$$
\begin{aligned}
& \sum_{d \leqslant x^{2}} \tau(d)^{B} \frac{\nu(d)}{d} \#\left\{\left(n_{1}, n_{2}\right) \in \mathcal{K} . x \cap \mathbf{Z}^{2}:\left(n_{1}, d\right) \neq 1, P^{-}\left(\left(n_{1}, d\right)\right)>y\right\} \\
\ll & \sum_{p>y} \frac{1}{p} \#\left\{\left(n_{1}, n_{2}\right) \in \mathcal{K} . x \cap \mathbf{Z}^{2}: p \mid n_{1}\right\} \sum_{d \ll x^{2}} \tau(d)^{B} \frac{\nu(d)}{d} \\
\ll & \frac{x^{2}}{y} \log (2 x)^{c(B)} .
\end{aligned}
$$


D'autre part, si $y$ est plus grand que les coefficients de $G$, on peut observer que les hypothèses $p \mid\left(n_{1}, G\left(n_{1}, n_{2}\right)\right)$ et $p>y$ impliquent $p \mid n_{2}$ et donc

$$
\begin{aligned}
& \sum_{d \leqslant x^{2}} \tau(d)^{B} \#\left\{\left(n_{1}, n_{2}\right) \in \mathcal{K} . x \cap \mathbf{Z}^{2}:\left(n_{1}, d\right) \neq 1, P^{-}\left(\left(n_{1}, d\right)\right)>y \text { et } d \mid G\left(n_{1}, n_{2}\right)\right\} \\
\ll & \sum_{\substack { p>y \\
\begin{subarray}{c}{n_{1}, n_{2} \leqslant x \\
p \mid\left(n_{1}, n_{2}\right){ p > y \\
\begin{subarray} { c } { n _ { 1 } , n _ { 2 } \leqslant x \\
p | ( n _ { 1 } , n _ { 2 } ) } }\end{subarray}} \tau\left(G\left(n_{1}, n_{2}\right)\right)^{B+1} \\
\ll & \frac{x^{2}}{y} \log (2 x)^{c(B)} .
\end{aligned}
$$

En observant que cette dernière estimation est aussi valable trivialement pour $y$ borné d'après le théorème 1 de [lBB06], on en déduit que 4.10 est vrai pour tout $y \geqslant 1$ ce qui achève la preuve.

Acknowledgement. L'auteur tient à remercier chaleureusement Régis de La Bretèche, Gérald Tenenbaum et Cécile Dartyge pour leur disponibilité et leurs conseils avisés autour de la rédaction de cet article.

\section{RÉFÉRENCES}

[All82a] K. Alladi, Asymptotic estimates of sums involving the Moebius function, J. Number Theory 14 (1982), no. 1, 86-98.

[All82b] Asymptotic estimates of sums involving the Moebius function. II, Trans. Amer. Math. Soc. 272 (1982), no. 1, 87-105.

[BBDT12] A. Balog, V. Blomer, C. Dartyge, and G. Tenenbaum, Friable values of binary forms, Comment. Math. Helv. 87 (2012), no. 3, 639-667.

[Bom76] E. Bombieri, The asymptotic sieve, Rend. Accad. Naz. XL (5) 1/2 (1975/76), 243-269 (1977).

[Dan99] S. Daniel, On the divisor-sum problem for binary forms, J. Reine Angew. Math. 507 (1999), 107-129.

[Ded78] R. Dedekind, Über den zusammenhang zwischen der theorie der ideale und der höheren kongruenzen, Abh. Kgl. Ges. Wiss. Göttingen 23 (1878), 1-23.

[dlBB06] R. de la Bretèche and T. D. Browning, Sums of arithmetic functions over values of binary forms, Acta Arith. 125 (2006), no. 3, 291-304.

[dlBB10] Le problème des diviseurs pour des formes binaires de degré 4, J. Reine Angew. Math. 646 (2010), 1-44.

[FI97] É. Fouvry and H. Iwaniec, Gaussian primes, Acta Arith. 79 (1997), no. 3, 249-287.

[FI98a] J. Friedlander and H. Iwaniec, Asymptotic sieve for primes, Ann. of Math. (2) 148 (1998), no. 3, 1041-1065.

[FI98b] The polynomial $X^{2}+Y^{4}$ captures its primes, Ann. of Math. (2) 148 (1998), no. 3, 945-1040.

[FI10] O Opera de cribro, American Mathematical Society Colloquium Publications, vol. 57, American Mathematical Society, Providence, RI, 2010.

[Gra08] A. Granville, Smooth numbers : computational number theory and beyond, Algorithmic number theory : lattices, number fields, curves and cryptography, Math. Sci. Res. Inst. Publ., vol. 44, Cambridge Univ. Press, Cambridge, 2008, pp. 267-323.

[Gre70] G. Greaves, On the divisor-sum problem for binary cubic forms, Acta Arith. 17 (1970), 1-28.

[Gre71]_ L L L L L _ prime factors of binary forms, J. Number Theory 3 (1971), 35-59. 
[Hel06] H. A. Helfgott, The parity problem for reducible cubic forms, J. London Math. Soc. (2) 73 (2006), no. 2, 415-435.

[Hil84] A. Hildebrand, On a problem of Erdös and Alladi, Monatsh. Math. 97 (1984), no. 2, 119-124.

[Hil86]_ On the number of positive integers $\leqslant x$ and free of prime factors $>y$, J. Number Theory 22 (1986), no. 3, 289-307.

[HR74] H. Halberstam and H.-E. Richert, Sieve methods, Academic Press [A subsidiary of Harcourt Brace Jovanovich, Publishers], London-New York, 1974, London Mathematical Society Monographs, No. 4.

[HT93] A. Hildebrand and G Tenenbaum, Integers without large prime factors, J. Théor. Nombres Bordeaux 5 (1993), no. 2, 411-484.

[HTW08] G. Hanrot, G. Tenenbaum, and J. Wu, Moyennes de certaines fonctions multiplicatives sur les entiers friables. II, Proc. Lond. Math. Soc. (3) 96 (2008), no. 1, 107-135.

[LT] A. Lachand and G. Tenenbaum, Notes sur les valeurs moyennes criblées de certaines fonctions arithmétiques, to appear in Quart. J. Math. Oxford Ser. (2).

[Mar06] G. Marasingha, On the representation of almost primes by pairs of quadratic forms, Acta Arith. 124 (2006), no. 4, 327-355.

[Pom08] C. Pomerance, Smooth numbers and the quadratic sieve, Algorithmic number theory : lattices, number fields, curves and cryptography, Math. Sci. Res. Inst. Publ., vol. 44, Cambridge Univ. Press, Cambridge, 2008, pp. 69-81.

[Sel52] A. Selberg, On elementary methods in primenumber-theory and their limitations, Den 11te Skandinaviske Matematikerkongress, Trondheim, 1949, Johan Grundt Tanums Forlag, Oslo, 1952, pp. 13-22.

[Ten90] G. Tenenbaum, Sur un problème d'Erdős et Alladi, Séminaire de Théorie des Nombres, Paris 1988-1989, Progr. Math., vol. 91, Birkhäuser Boston, Boston, MA, 1990, pp. 221-239.

[Ten08] _ Introduction à la théorie analytique et probabiliste des nombres, 3ième édition ed., Échelles, Belin, 2008.

A. Lachand : Institut Élie Cartan, Université de Lorraine 1, B.P. 70239, 54506 Vandeeuvre-Lès-Nancy Cedex, France

E-mail address: armand.lachand@univ-lorraine.fr 